\title{
Erratum: Spatial and temporal propagation of Kondo correlations [Phys. Rev. B 90, 045117 (2014)]
}

Benedikt Lechtenberg and Frithjof B. Anders

(Received 5 January 2015; published 21 January 2015)

\author{
DOI: 10.1103/PhysRevB.91.039907 PACS number(s): 03.65.Yz, 73.21.La, 73.63.Kv, 76.20.+q, 99.10.Cd
}

Figure 17 in Appendix A of our paper shows the wrong graph. Instead of the correct figure (depicted below) Figs. 13(a) and 13(b) have been printed a second time. The contents of the paper and all conclusions remain unaffected.

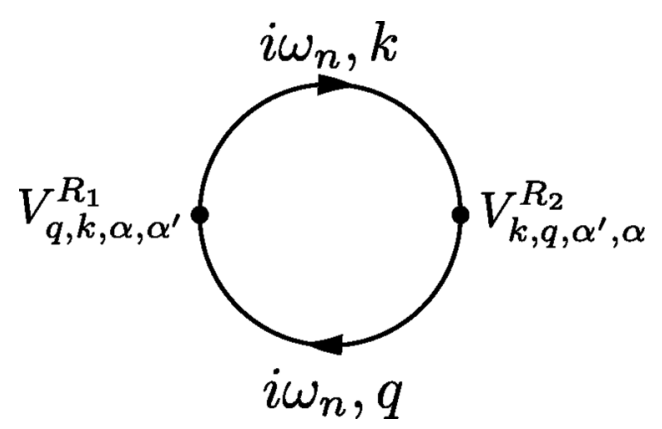

FIG. 17. The second-order Feynman diagram generating the lowest-order contribution to the RKKY interaction between two localized spins mediated by a spin excitation propagating through the metallic host. 